\title{
The Photometric Maximum in Cosmicflows-2
}

\author{
Lorenzo Zaninetti \\ Physics Department, via P. Giuria 1, Turin, Italy \\ Email: zaninetti@ph.unito.it
}

How to cite this paper: Zaninetti, L. (2018) The Photometric Maximum in Cosmicflows-2. Journal of High Energy Physics, Gravitation and Cosmology, 4, 123-131. https://doi.org/10.4236/jhepgc.2018.41011

Received: November 28, 2017

Accepted: January 28, 2018

Published: January 31, 2018

Copyright $\odot 2018$ by author and Scientific Research Publishing Inc. This work is licensed under the Creative Commons Attribution International License (CC BY 4.0).

http://creativecommons.org/licenses/by/4.0/

\begin{abstract}
Based on well known photometric rules for the number of galaxies as function of the distance in Mpc we model the so called "Great Wall" which is visible on the Cosmicflows-2 catalog. The gravitational field is evaluated at the light of the shell theorem and a finite value for the gravitational field is numerically derived.
\end{abstract}

\section{Keywords}

Cosmology, Observational Cosmology, Distances, Redshifts, Radial Velocities, Spatial Distribution of Galaxies, Magnitudes and Colors, Luminosities

\section{Introduction}

Before to start we briefly review the Hubble law, after [1], which is a linear relationship between expansion velocity and distance in

$$
V=H_{0} D=c z,
$$

where $H_{0}$ is the Hubble constant $H_{0}=100 \mathrm{~h} \mathrm{~km} \cdot \mathrm{s}^{-1} \cdot \mathrm{Mpc}^{-1}$, with $h=1$ when $h$ is not specified, $D$ is the distance in Mpc, $c$ is the light velocity and $z$ is the redshift. As an example a recent evaluation, see [2], quotes

$$
H_{0}=(74.4 \pm 3) \mathrm{km} \cdot \mathrm{s}^{-1} \cdot \mathrm{Mpc}^{-1} .
$$

The original Hubble's law was based on $\approx 20$ galaxies of which was known the distance and the velocity; conversely in the last years the number of catalogs for galaxies with redshift available for public downloading has progressively grown. This huge amount of data allows setting up critical tests between different theoretical models over the various aspects of the Large Scale Structures. The layout of the paper is as follows. In Section 2, we describe some representative catalogs of galaxies. In Section 3, we introduce the adopted luminosity function for galaxies. In Section 4, we model the maximum in the number of galaxies as func- 
tion of the distance. In Section 5, we evaluate the gravitational field as produced by the visible galaxies of Cosmicflows- 2 catalog. We conclude in Section 6 .

\section{The Catalogs of Galaxies}

A first kind of catalog for galaxies is represented by those focused as slice as the two-degree Field Galaxy Redshift Survey, in the following 2dFGRS, see [3], or the Sloan Digital Sky Survey (SDSS), see [4]. A second classification is about the all-sky catalogs such as the 2MASS Redshift Survey (2MRS), see [5], or the Cosmicflows-2, see [2]. As a first example a strip of the 2dFGRS is shown in Figure 1. The below Figure, which is a $3^{\circ}$ slice, shows that the galaxies resides on filaments rather to be distributed in a uniform $2 \mathrm{D}$ way. A first extrapolation allows to state that the galaxies are disposed on the surface of bubbles rather than to fill uniformly the 3D space. The statistics of the voids is a topic of research and an important parameter is the average radius of the voids, $\langle R\rangle:$ [6] quotes $\langle R\rangle=18.23 h^{-1} \mathrm{Mpc}$ and Table 1 in [7] quotes a variable average radius ranging from $\langle R\rangle=15.964 h^{-1} \mathrm{Mpc}$ to $\langle R\rangle=63.347 h^{-1} \mathrm{Mpc}$ according to the selected sample. As second example is represented by the overall Cosmicflows- 2 catalog, data available at http://vizier.u-strasbg.fr/viz-bin/VizieR, and Figure 2 reports the sky distribution of such a catalog in Galactic coordinates.

Also in this case the voids are can be visualized selecting a thin $(20 \mathrm{Mpc})$ spherical shell, see Figure 3.

\section{Luminosity Function for Galaxies}

The distance modulus is

$$
m-M=5 \log (d)-5,
$$

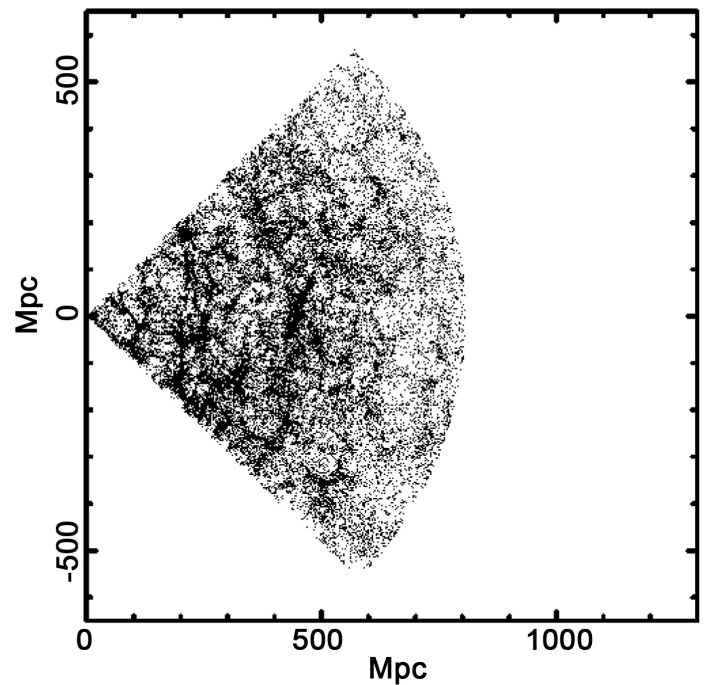

Figure 1. Cone-diagram of the galaxies in the 2dFGRS with distance $\in[4 \mathrm{Mpc}, 470 \mathrm{Mpc}]$. This plot contains 117,293 galaxies. 


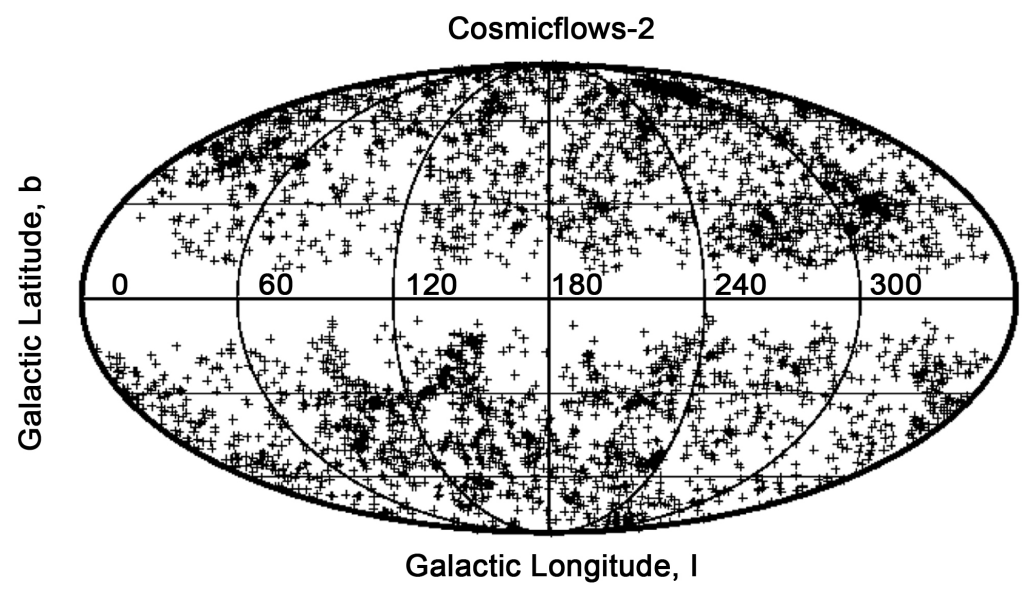

Figure 2. Sky distribution of 4970 Cosmicflows-2's galaxies in galactic coordinates projected using the Mollweide projection.

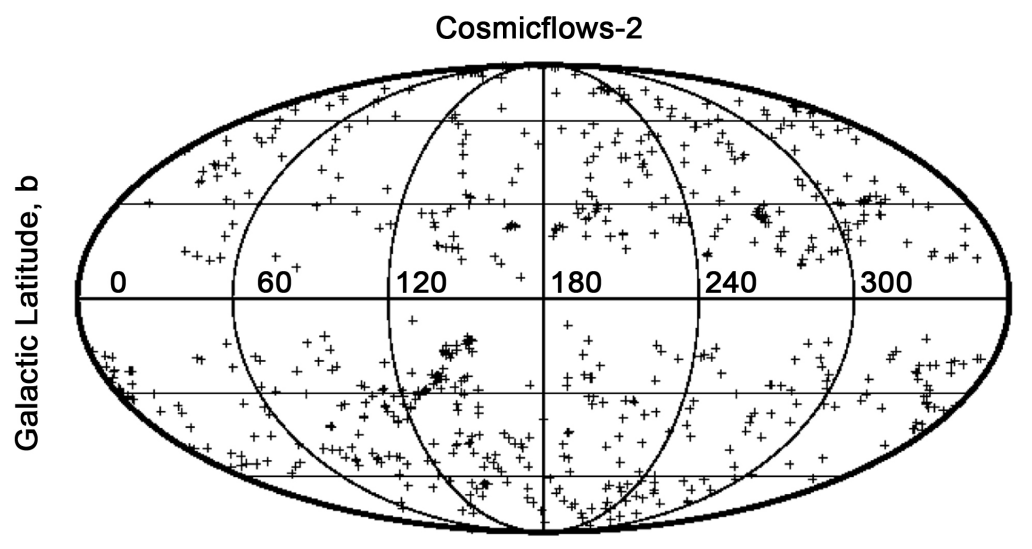

Galactic Longitude, I

Figure 3. Sky distribution of 713 Cosmicflows-2's galaxies with distance $\in$ [63.8 Mpc, 83.8 Mpc].

where $m$ is the apparent magnitude, $M$ is the absolute magnitude and $d$ is the distance in pc.

Let $L$, the luminosity of a galaxy, be defined in $[0, \infty]$. The Schechter LF of galaxies, $\Phi$, see $[8]$, is

$$
\Phi\left(L ; \Phi^{*}, \alpha, L^{*}\right) \mathrm{d} L=\left(\frac{\Phi^{*}}{L^{*}}\right)\left(\frac{L}{L^{*}}\right)^{\alpha} \exp \left(-\frac{L}{L^{*}}\right) \mathrm{d} L,
$$

where $\alpha$ sets the slope for low values of $L, L^{*}$ is the characteristic luminosity, and $\Phi^{*}$ represents the number of galaxies per $\mathrm{Mpc}^{3}$. The normalization is

$$
\int_{0}^{\infty} \Phi\left(L ; \Phi^{*}, \alpha, L^{*}\right) \mathrm{d} L=\Phi^{*} \Gamma(\alpha+1),
$$

where

$$
\Gamma(z)=\int_{0}^{\infty} \mathrm{e}^{-t} t^{z-1} \mathrm{~d} t
$$

is the Gamma function. The average luminosity, $\langle L\rangle$, is 


$$
\left\langle\Phi\left(L ; \Phi^{*}, \alpha, L^{*}\right)\right\rangle=L^{*} \Phi^{*} \Gamma(\alpha+2) .
$$

An equivalent form in absolute magnitude of the Schechter LF is

$$
\Phi\left(M ; \Phi^{*}, \alpha, M^{*}\right) \mathrm{d} M=0.921 \Phi^{*} 10^{0.4(\alpha+1)\left(M^{*}-M\right)} \exp \left(-10^{0.4\left(M^{*}-M\right)}\right) \mathrm{d} M,
$$

where $M^{*}$ is the characteristic magnitude.

A typical result of the Schechter LF in the case of Cosmicflows- 2 is reported in Figure 4.

\section{The Photometric Maximum}

The flux, $f$ is

$$
f=\frac{L}{4 \pi r^{2}},
$$

where $r$ is the distance and $L$ the luminosity of the galaxy. The joint distribution in distance, $r$, and flux, $f$, for the number of galaxies is

$$
\frac{\mathrm{d} N}{\mathrm{~d} \Omega \mathrm{d} r \mathrm{~d} f}=\frac{1}{4 \pi} \int_{0}^{\infty} 4 \pi r^{2} \mathrm{~d} r \Phi\left(\frac{L}{L^{*}}\right) \delta\left(f-\frac{L}{4 \pi r^{2}}\right),
$$

were the factor $\left(\frac{1}{4 \pi}\right)$ converts the number density into density for solid angle and the Dirac delta function selects the required flux. In the case of Schechter LF of galaxies the number of galaxies as function of the distance is

$$
\frac{\mathrm{d} N}{\mathrm{~d} \Omega \mathrm{d} r \mathrm{~d} f}=\frac{1}{L^{*}} 4 \pi r^{4} \Phi^{*}\left(4 \frac{\pi f r^{2}}{L^{*}}\right)^{\alpha} \mathrm{e}^{-4 \frac{\pi r^{2}}{L^{*}}} .
$$

We now introduce the critical radius $r_{\text {crit }}$

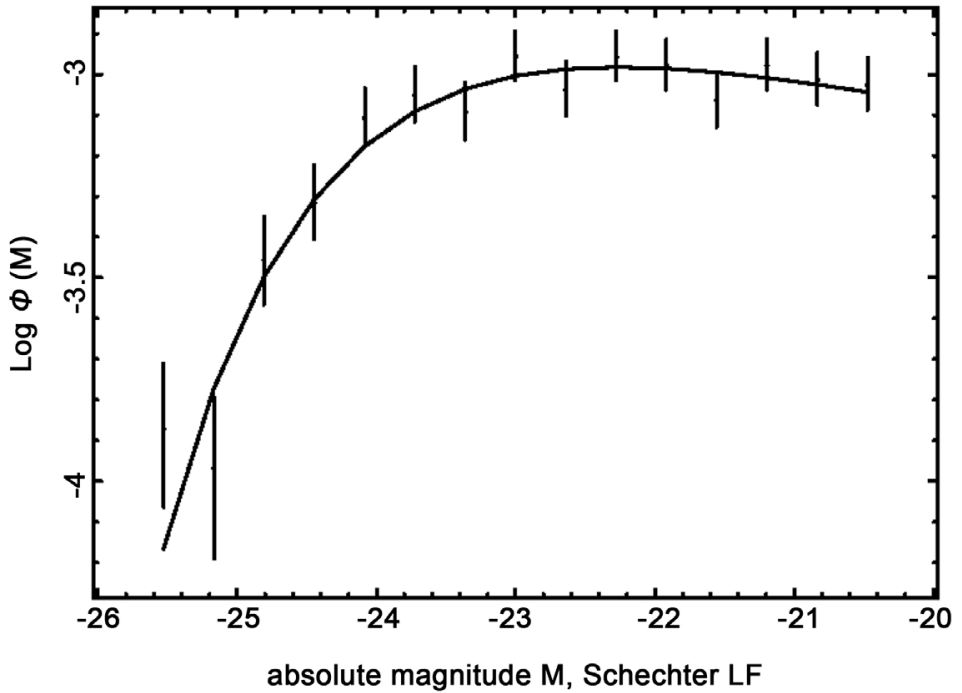

Figure 4. The observed Schechter LF for galaxies of Cosmicflows-2, empty stars with error bar, and the fit by the Schechter LF when

$P h i^{*}=0.0018 h^{3} \mathrm{Mpc}^{-3}, M^{*}=-24.2-5 \log _{10}(h)$ and $\alpha=-0.83$. 


$$
r_{\text {crit }}=\frac{1}{2} \frac{\sqrt{L^{*}}}{\sqrt{\pi} \sqrt{f}} .
$$

Therefore the joint distribution in distance and flux becomes

$$
\frac{\mathrm{d} N}{\mathrm{~d} \Omega \mathrm{d} r \mathrm{~d} f}=\frac{1}{L^{*}} 4 \pi r^{4} \Phi^{*}\left(\frac{r^{2}}{r_{\text {crit }}^{2}}\right)^{\alpha} \mathrm{e}^{-\frac{r^{2}}{r_{\text {crit }}^{2}}} .
$$

The above number of galaxies has a maximum at $r=r_{\max }$ :

$$
r_{\max }=\sqrt{2+\alpha} r_{\text {crit }} \text {, }
$$

and the average distance of the galaxies, $\langle r\rangle$, is

$$
\langle r\rangle=\frac{r_{\text {crit }} \Gamma(3+\alpha)}{\Gamma\left(\frac{5}{2}+\alpha\right)} .
$$

Figure 5 presents the number of galaxies observed in Cosmicflows-2 as a function of the distance for a given window in flux, as well as the theoretical curve. The luminosity of a galaxy is produced and attenuated according to the electromagnetism. We now deal with the mass of a galaxy. A transformation of the luminosity of a galaxy, $L$, by the mass, $\mathcal{M}$, is given by the nonlinear Formula (9) in [9]

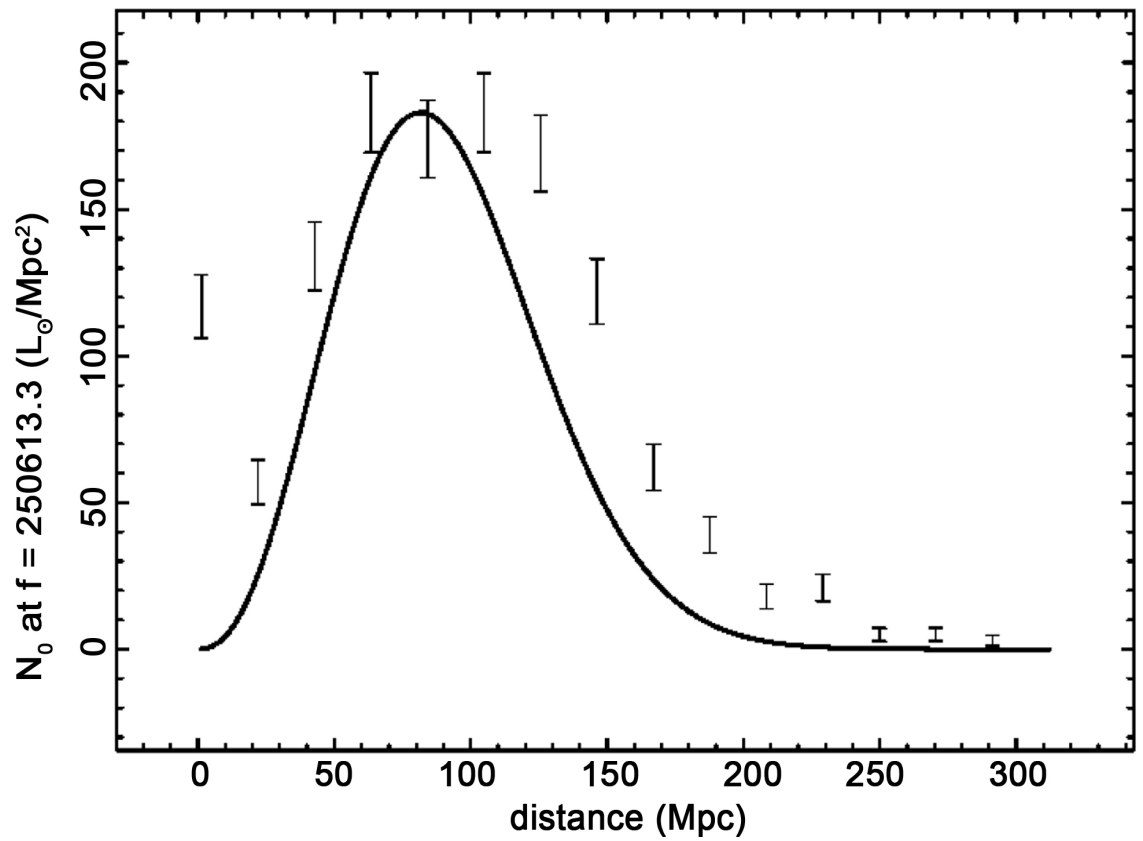

Figure 5. The galaxies of Cosmicflows-2 with $12498.8 L_{\odot} / \mathrm{Mpc}^{2} \leq f \leq 488727.8 L_{\odot} / \mathrm{Mpc}^{2}$ are organized by frequency versus distance, (empty circles); the error bar is given by the square root of the frequency. The maximum frequency of the observed galaxies is at $d=73.8$ Mpc. The full line is the theoretical curve generated by $\frac{\mathrm{d} N}{\mathrm{~d} \Omega \mathrm{d} r \mathrm{~d} f}$ as given by the application of the Schechter LF which is Equation (11) and the theoretical maximum is at $d=81.69 \mathrm{Mpc}$. The parameters are $L^{*}=1.8 \times 10^{10} L_{\odot}$ and $\alpha=-0.83$. 


$$
\frac{\mathcal{M}}{L}=2.35\left(\frac{L}{10^{10} L_{\odot}}\right)^{0.32}
$$

We pay careful attention to the interval of existence in absolute magnitude versus distance for Cosmicflows-2 catalog, see Figure 6.

The progressive decrease of the interval of existence for the absolute magnitude is known as Malmquist bias [10]. Conversely the mass of a galaxy does not disappear with distance and therefore produce the gravitational field even if the galaxy is not visible due to the instrumental limitations.

\section{Gravitational Forces}

The shell theorem, according to proposition LXX, theorem XXX, in Principia [11] states that "If to every point of a spherical surface there tend equal centripetal forces decreasing in the square of the distances from those points, I say, that a corpuscle placed within that superficies will not be attracted by those forces any way" or more simply "The gravitational field inside a uniform spherical shell is zero" [12] [13] [14]. The galaxies are thought to reside on irregular shells which are approximated by a given radius. Due to the fact that the shapes of the above shells are neither exactly spherical nor uniformly populated by galaxies we search for a low value of the gravitational field rather than the theoretical zero. We therefore analyze a $2 \mathrm{D}$ box with size of $30 \mathrm{Mpc}$ and masses as given by formula 16. The forces in every point of the considered box are evaluated according to the Newtonian force where $G$, the Newtonian constant of gravitation, is

$$
G=4.49975 \times 10^{-6} \mathrm{Mpc}^{3} / M_{\text {gal }} \cdot \mathrm{yr}^{2},
$$

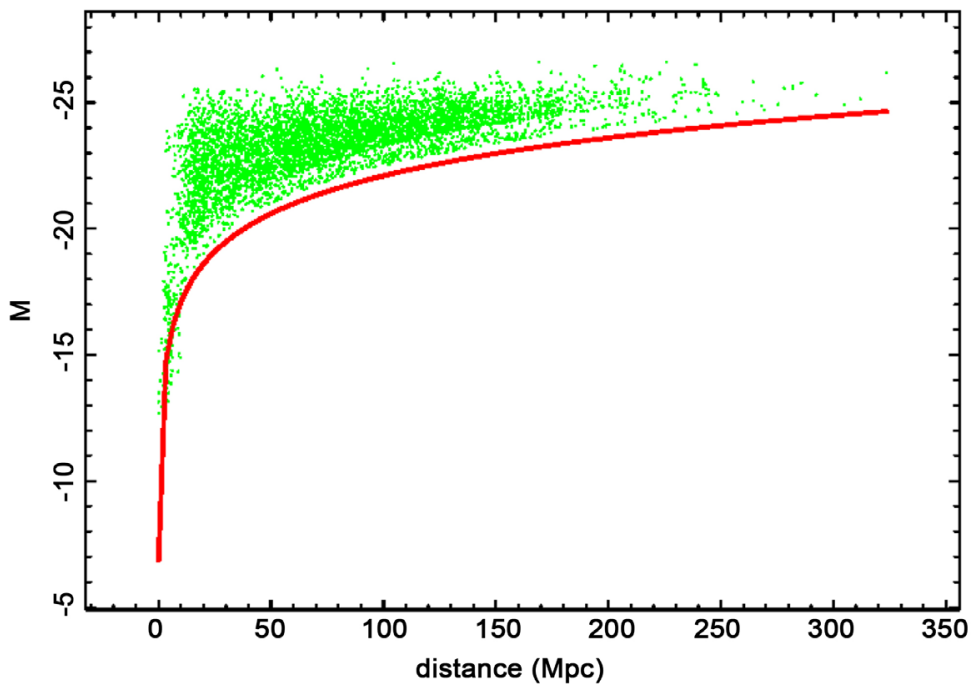

Figure 6. The absolute magnitude $M$ of 4970 galaxies belonging to Cosmicflows- 2 when the absolute bolometric magnitude is 3.39 and

$H_{0}=74.4 \mathrm{~km} \cdot \mathrm{s}^{-1} \cdot \mathrm{Mpc}^{-1}$ (green points). The upper theoretical curve, Equation (3), is reported as a red thick line when $m=15.39$. 
where the length is in $\mathrm{Mpc}$, mass is in $M_{\text {gal }}$ which are $10^{11} M_{\odot}$ and yr 8 are $10^{8} \mathrm{yr}$, see more details in [15]. The masses do not disappear with distance and the above value of the box allows processing a complete sample of galaxies. Figure 7 reports the values of the gravitational field as a cut in the middle of the $2 \mathrm{D}$ box. Figure 8 reports the values of the gravitational field organized as a two color map.

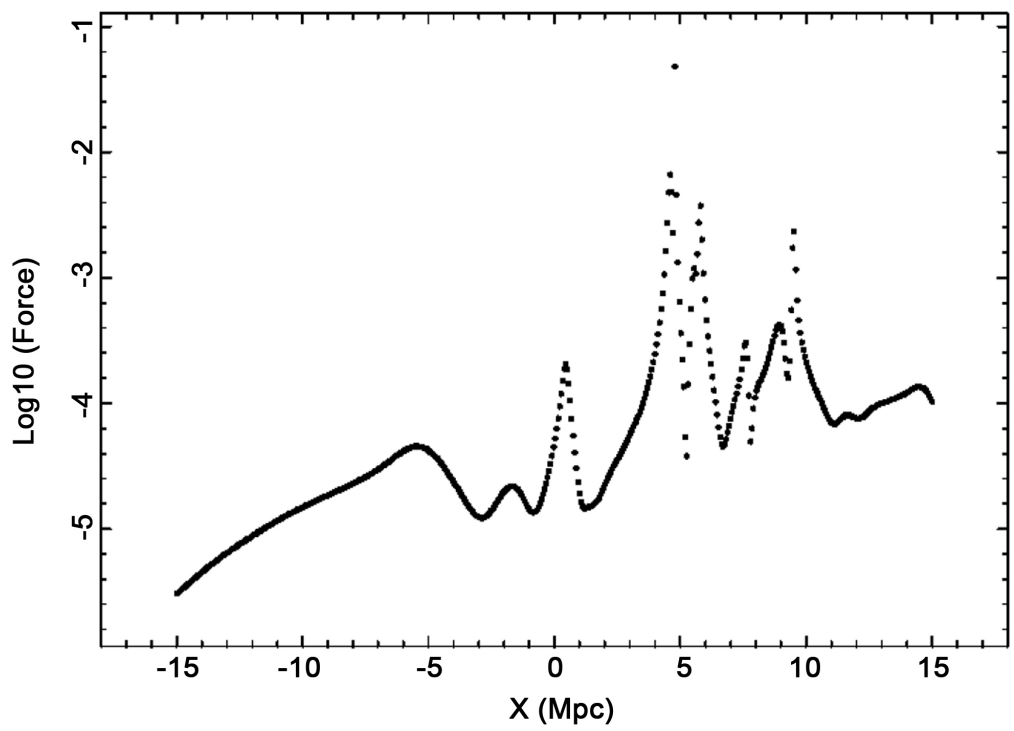

Figure 7. Cut-line of the $2 \mathrm{D}$ gravitational forces expressed in $\mathrm{Mpc} \cdot M_{\text {gal }} / \mathrm{yr}^{2}$ (decimal logarithm).

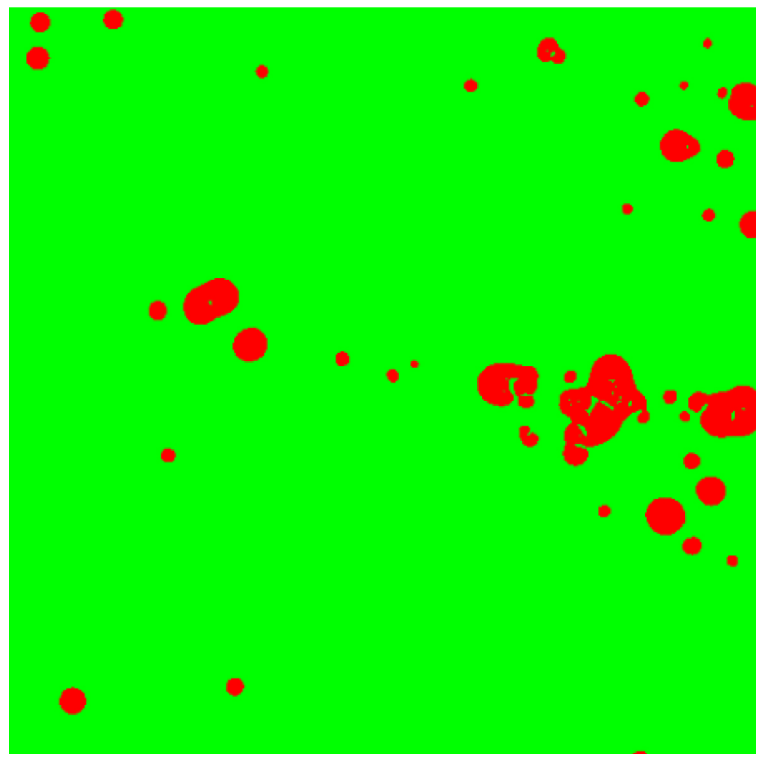

Figure 8. Color scheme for the values of the gravitational field evaluated in $500 \times 500$ points. The red zone has values of gravitational field in the interval $\left[4.27 \mathrm{Mpc} \cdot M_{\text {gal }} / \mathrm{yr}^{2}, 0.00017 \mathrm{Mpc} \cdot M_{\text {gal }} / \mathrm{yr}^{2}{ }^{2}\right]$ (the zones near the galaxies) and the green zone has values of gravitational field in the interval $\left[0.00017 \mathrm{Mpc} \cdot M_{\text {gal }} / \mathrm{yr}^{2}, 1.7 \times 10^{-8} \mathrm{Mpc} \cdot M_{\text {gal }} / \mathrm{yr}^{2}\right]$ (the zones of the voids). 


\section{Conclusion}

The observed "great wall" is theoretically explained by a maximum in the number of galaxies as function of the distance in $\mathrm{Mpc}$, see Figure 5. The two concepts of repeller and attractor, see [16], are not used in our analysis. The presence of voids approximated by spheres in the spatial distribution of galaxies allows analyzing the gravitational field at the light of the shell theorem. Due to the facts that the number of galaxies is finite and the masses of galaxies are distributed according to a gamma probability density function the gravitational field at the center of the voids turns out to be $\approx 2 \times 10^{-8} \mathrm{Mpc} \cdot M_{\text {gal }} / \mathrm{yr}^{2}$ rather than zero.

\section{Acknowledgements}

This research has made use of the VizieR catalogue access tool, CDS, Strasbourg, France.

\section{References}

[1] Hubble, E. (1929) A Relation between Distance and Radial Velocity among Extra-Galactic Nebulae. Proceedings of the National Academy of Science, 15, 168-173. https://doi.org/10.1073/pnas.15.3.168

[2] Tully, R.B., Courtois, H.M., Dolphin, A.E., et al. (2013) Cosmicflows-2: The Data. Astronomical Journal, 146, 86. https://doi.org/10.1088/0004-6256/146/4/86

[3] Colless, M., Dalton, G., Maddox, S., et al. (2001) The 2dF Galaxy Redshift Survey: Spectra and Redshifts. Monthly Notices of the Royal Astronomical Society, 328, 1039-1063. https://doi.org/10.1046/j.1365-8711.2001.04902.x

[4] Berlind, A.A., Frieman, J., Weinberg, D.H., et al. (2006) Percolation Galaxy Groups and Clusters in the SDSS Redshift Survey: Identification, Catalogs, and the Multiplicity Function. Astrophysical Journal Supplement Series, 167, 1-25.

https://doi.org/10.1086/508170

[5] Huchra, J.P., Macri, L.M., Masters, K.L., et al. (2012) The 2MASS Redshift Survey-Description and Data Release. Astrophysical Journal Supplement Series, 199, 26. https://doi.org/10.1088/0067-0049/199/2/26

[6] Pan, D.C., Vogeley, M.S., Hoyle, F., Choi, Y.Y. and Park, C. (2012) Cosmic Voids in Sloan Digital Sky Survey Data Release 7. Monthly Notices of the Royal Astronomical Society, 421, 926-934. https://doi.org/10.1111/j.1365-2966.2011.20197.x

[7] Mao, Q., Berlind, A.A., Scherrer, R.J., et al. (2017) A Cosmic Void Catalog of SDSS DR12 BOSS Galaxies. Astrophysical Journal, 835, 161. https://doi.org/10.3847/1538-4357/835/2/161

[8] Schechter, P. (1976) An Analytic Expression for the Luminosity Function for Galaxies. Astrophysical Journal, 203, 297-306. https://doi.org/10.1086/154079

[9] Cappellari, M., Bacon, R., Bureau, M., Damen, M.C., et al. (2006) The SAURON project-IV. The Mass-to-Light Ratio, the Virial Mass Estimator and the Fundamental Plane of Elliptical and Lenticular Galaxies. Monthly Notices of the Royal Astronomical Society, 366, 1126-1150. https://doi.org/10.1111/j.1365-2966.2005.09981.x

[10] Malmquist, K.G. (1920) A Study of the Stars of Spectral Type A. Meddelanden fran Lunds Astronomiska Observatorium Series II, 22, 1.

[11] Newton, I. (1687) Philosophiae Naturalis Principia Mathematica. Jussu Societatis 
Regiae ac Typis Josephi Streater, London.

[12] Arens, R. (1990) Newton's Observations about the Field of a Uniform Thin Spherical Shell. Note di Matematica, 10, 39.

[13] Chandrasekhar, S. (1995) Newton' Principia for the Common Reader. Clarendon, Oxford.

[14] Borghi, R. (2014) On Newton's Shell Theorem. European Journal of Physics, 35, Article ID: 028003. https://doi.org/10.1088/0143-0807/35/2/028003

[15] Zaninetti, L. (2012) The Intergalactic Newtonian Gravitational Field and the Shell Theorem. Serbian Astronomical Journal, 185, 17-23.

[16] Hoffman, Y., Pomarède, D., Tully, R.B. and Courtois, H.M. (2017) The Dipole Repeller. Nature Astronomy, 1, Article No. 0036. 